gekocht. Die eingedampften Filtrate schieden bei weiterer freiwilliger Verdunstung allmählich, neben einigen farblosen, nicht näher untersuchten Kryställchen, gelbe, dem Rutin im Aeußern und in der Art der Abscheidung entsprechende krystallinische Massen aus. Nach dem Umkrystallisieren aus heißem Wasser stimmten dieselben, mit Rutin direkt verglichen, sowohl im Schmelzpunkt, als auch in den Reaktionen vollständig überein. Die gleiche Uebereinstimmung war bei der daraus dargestellten Acetylverbindung zu konstatieren, so daß, unter Berücksichtigung der von $\mathrm{R}$. T i e $\mathrm{m}$ a $\mathrm{n} n$ gemachten Beobachtungen, wohl an der Identität des aus Globularia Alypum isolierten gelben Farbstoffes mit Rutin nicht zu zweifeln ist.

Die Mutterlaugen von den Rutinausscheidungen lieferten nach dem Ansäuern und Ausschütteln mit Aether eine sehr kleine Menge von nadelförmigen Krystallen, wolche die Reaktionen der Protokatechusäu re zeigten. Eine weitere Identifizierung gestattete leider die geringe Menge dieser Säure nicht.

\title{
Ueber das Sakuranin, ein neues Glykosid der Rinde von Prunus Pseudo-Cerasus Lindl. var. Sieboldi Maxim.
}

\author{
Von Y. As a h in a, \\ Assistent am Pharmazeutischen Institut der Kaiserlichen \\ Universität Tokyo (Japan).
}

(Eingegangen den 22. III. 1908.)

Es ist schon längst bekannt, daß in einigen Pomaceen und Amygdalaceen ein stickstofffreies Glykosid, Phloridzin, sich befindet.

Der japanische Kirschbaum wurde, bis zur jüngsten Zeit nur wenig untersucht. Diese Pflanze, japanisch $\mathrm{S}$ a $\mathrm{k}$ u $\mathrm{r}$ a genannt, kommt in drei Varietäten vor: Prunus Pseudo-Cerasus Lindl.

var. Spontanea Maxim. = Yama-Sakura;

var. Sieboldi Maxim. = Yoshino-Sakura;

var. Hortensis Maxim. = Yaë-Sakura.

Die erste Varietät ist der Bewohner von Bergen und Wäldern, während die zwei letzteren am häufigsten als allerliebste Zierpflanze in den Gärten kultiviert werden.

Die Blüten und Blätter des japanischen Kirschbaumes entwickeln beim Aufbewahren einen Wohlgeruch, weleher im frischen 
Zustande sich nicht erkennen läßt. Sie finden daher in Japan als Gewürze eine beschränkte Anwendung. $\mathrm{Zu}$ diesem Zwecke werden sie mit Kochsalz vermischt und unter Druck in feuchtem Zustande einige Zeitlang aufbewahrt. Die so zubereiteten Blüten werden in heißes Wasser eingetragen und wie Tee genossen; die Blätter werden zum Parfümieren von Kuchen (Sakura-Mochi) benutzt.

Im Jahre 1891 hatte Herr Prof. Dr. $\mathrm{N}$ a $\mathrm{g} \mathrm{a}^{1}$ ) darin Cumarin nachgewiesen und bestätigte, daß der Riechstoff in den frisch abgepflückten Blättern erst nach 12-24 Stunden gebildet wird. Nach ihm sollen die Blätter außer Cumarin noch Cyanwasserstoff liefern.

Im Jahre 1904 beschäftigte sich Herr S. H a n z a w a mit der Untersuchung der Sakurarinde und hatte darin ein Glykosid gefunden. Da die Untersuchung leider abgebrochen wurde, so unterzog jch mich, auf Veranlassung von Herrn Professor Dr. Shimo y a ma, der Aufgabe eine weitere Untersuchung dieses Glykosids auszuführen. Bevor jch auf die Ergebnisse meiner eigenen Arbeit näher eingehe, sei es mir gestattet, hier über die Resultate von Herrn $\mathrm{Hanzaw} \mathrm{a}^{2}$ ) kurz mitzuteilen, da er seine Arbeit noch in keiner Zeitschrift publiziert hat.

Zur Darstellung dieses Glykosids kochte er die frisch abgeschälte, zerkleinerte Rinde mit Wasser aus. Der Auszug wurde alsdann durch Abdampfen eingeengt und hierauf, zur Beseitigung der darin in reichlicher Menge vorhandenen Gerbstoffe und anderen Verunreinigungen, mit frisch gefälltem Aluminiumhydroxyd geschüttelt und noch heiß filtriert. Aus dem bräunlich gefärbten Filtrat schied sich beim Erkalten ein flockiger, in feinen Nadeln krystallisierter Niederschlag ab. Letzteren krystallisierte $\mathrm{Hanza}$ a wiederholt aus kochendem Wasser oder Alkohol um. Die so erhaltenen reinen Krystalle stellen weiße, leichte Nadeln dar; dieselben sollen bei $87^{\circ}$ erweichen, sich gegen $150^{\circ}$ gelb färben, um endlich bei $200^{\circ}$ zu einer zähen Masse zu schmelzen. Die Krystalle sind in Alkohol leicht löslich, in heißem Wasser schwer, in Aether kaum löslich. Die alkoholische oder wässerige Lösung wird durch Eisenchlorid gelbbraun gefärbt. Beim Kochen mit verdünnten Mineralsäuren spalten die Krystalle sich in eine krystallinische, schwer lösliche Substanz und Zucker. Dieses

1) Journal of the Pharmaceutical Society of Japan 1891, S. 1090 (japanisch).

2) Privatmitteilung von Herrn $S$. $\mathrm{Hanz}$ a wa. 
krystallinische Spaltungsprodukt ist in Aether und Alkohol leicht löslich, und gibt die alkoholische Lösung mit Fisenchlorid eine violette Färbung. Aus diesen Eigenschaften schloß $\mathrm{H}$ a n $\mathrm{z}$ a w a, daß die von ihm aus Sakurarinde isolierte Substanz ein Glykosid sei, welches mit dem Phloridzin nicht identisch ist.

\section{Darstellung des Glykosids.}

Seit drei Jahren habe ich mich mit der Sakurarinde beschäftigt, doch wegen des geringen Gehalts an Glykosid war ich bisher nicht imstande eine genügende Menge für eingehendere Untersuchungen darzustellen. Im letzten Frühjahr wurde mir jedoch ein größeres Quantum der Rinde von Prunus Pseudo-Cerasus var. Sieboldi zur Verfügung gestellt, und habe ich damit die Darstellung des Glykosids in etwas größerem Maßstabe ausgeführt.

Die zerkleinerte Rinde wurde zweimal mit kochendem Wasser, worin etwas Calciumkarbonat suspendiert war, ausgezogen, und der Auszug zuerst auf freiem Feuer, dann auf dem Wasserbade eingedampft, bis er beim lirkalten zu einem dicken Extrakt erstarrte. Das auf diese Weise gewonnene Extrakt bildete eine dunkelbraune Masse von bitterlich-zusammenziehendem Geschmack.

Je etwa $300 \mathrm{~g}$ des Extrakts wurden alsdann mit der 10 fachen Menge Wasser ausgekocht und wurde hierauf die Abkochung, deren Tomperatur noch über $90^{\circ}$ betrug, mit $50 \mathrm{ccm}$ Basisch-Aluminiumacetatlösung (Liquor aluminii acetici. Ph. G. III) versetzt. Sofort entstand ein reichlicher schmutzig brauner Niederschlag, und nach cinigen Minuten erschion die darüber befindliche Wasserschicht ganz klar. Dann wurde das Gemisch möglichst schnell durch ein mit heißem Wasser benetztes Faltenfilter abfiltriert. Das klare Filtrat, welchos goldgelb bis gelbbraun gefärbt war, wurde etwa 2 Tage lang zur Krystallisation stehen gelassen. Der hierbei ausgeschiedene flockige oder zu Krusten vereinigte Niederschlag wurde auf einem Tuch gesammelt, mit kaltem Wasser wiederholt gewaschen und langsam getrocknet. Die Rohkrystalle waren schon ziemlich weiß, aber mit mehr odor weniger Aschensubstanz, besonders mit Gips, verunreinigt.

Zur Reinigung derselben wurden sie in $50-60 \%$ igem Alkohol gelöst und das Filtrat mit so viel Wasser versetzt, bis eine bleibende Trübung eintrat. Nach einiger Zeit wurden hierauf die ausgeschiedenen, gelblich gefärbten Krystallkrusten abfiltriert. Da die alkoholische Lösung derselben durch Zusatz von Eisenchlorid eine bräunlich-violette Fürbung annahm, so ging hieraus 
hervor, daß dem Rohglykosid noch etwas von seinem Spaltungsprodukt beigemengt war. Zur weiteren Reinigung wurde die Substanz daher zweimal aus kochendem, absolutem Alkohol oder aus mit Wasser gesättigtem Essigäther umkrystallisiert. Die Mutterlauge wurde dann mit Vorteil zur Darstellung des Spaltungsproduktes verwendet.

Bezüglich der Ausbeute an Glykosid kann ich keine genauen Angaben machen, da der Gehalt an Glykosid in der Rinde nur sehr gering war, so daß die direkte Darstellung aus der Rinde immer nur eine Spur von Glykosid ergab. Aus je $100 \mathrm{~g}$ des Extrakts erhielt ich $1-3 \mathrm{~g}$ Rohglykosid; im ganzen betrug die Menge der Rohkrystalle etwa $100 \mathrm{~g}$, welche $\mathrm{zu}$ der vorliegenden Untersuchung verbraucht wurden.

Ich habe das Glykosid Sak u r a n in benannt.

\section{Elgenschaften des Sakuranins.}

Das Sakuranin ist stickstofffrei; dasselbe krystallisiert in feinen Nadeln von glänzend weißer Farbe und bitterem Geschmack. Es ist sehr leicht löslich in 50-60\% igem Alkohol und Pyridin, dagegen schwerer löslich in stärkerem Alkohol. In kaltem Wasser und Aether ist es fast unlöslich. Kochender absoluter Alkohol und mit Wasser gesättigter Essigäther nehmen es etwas auf und eignen sich am besten zur Reinigung desselben. Aus den zwei letztgenannten Lösungsmitteln gewonnene Krystalle sind krystallwasserfrei und schmelzen bei $210-212^{\circ}$. Wird das wasserfreie Sakuranin in verdünnten Alkohol gelöst und die Lösung mit so viel Wasser versetzt, bis eine bleibende Trübung eintritt, so wird daraus wasserhaltiges Sakuranin in Form von feinen Nadeln ausgefällt. Diese Krystalle sintern gegen $190^{\circ}$ zusammen und schmelzen bei $207^{\circ}$. Bei längerem Erhitzen auf $100^{\circ}$ geben dieselben alles Krystallwasser ab. Dic so erhaltene wasserfreie Substanz schmilzt dann etwas niedriger, nämlich bei $195^{\circ}$, jedoch verwandelt sich bei der nochmaligen Umkrystallisation aus absolutem Alkohol die niedriger schmelzende Modifikation wieder in das bei 210 bis $212^{\circ}$ schmelzende Sakuranin. In Alkalien, sowie in Ammoniak löst sich Sakuranin mit intensiv gelber Farbe; aus diesen Lösungen wird das Glykosid durch verdünnte Säuren, wie auch durch Kohlensäure, wieder krystallinisch abgeschieden. Beim Kochen mit verdünnten Mineralsäuren liefert das Sakuranin eine in Wasser schwer lösliche, krystallinische Substanz und eine, die Fehling'sche Lösung stark reduzierende Flüssigkeit. Die alkoholische Iösung 
des Sakuranins lenkt das polarisierte Licht nach links ab, und wird durch Eisenchlorid rein gelb gefärbt. Konzentrierte Schwefelsäure färbt das Glykosid intensiv braun und gibt eine gelbe Lösung. Wird das Sakuranin mit rauchender Salpetersäure zusammengebracht, so gibt es anfangs eine schmutzig grüne, nach einiger Zeit in Indigoblau übergehende Färbung. Erhitzt man Sakuranin mit Natriumamalgam und Wasser, und filtriert, so gibt das Filtrat mit Salzsäure einen flockigen Niederschlag, welcher in Alkohol mit roter Farbe löslich ist. Alkoholische oder wässerige Lösung des Sakuranins wird durch Bleiessig nicht gefällt.

Ana lyse des Sakuranins.

Bei $100^{\circ}$ getrocknetes Sakuranin wurde mit Kupferoxyd im geschlossenen Rohr verbrannt.

1. $0,3446 \mathrm{~g}$ lieferten $0,7381 \mathrm{~g} \mathrm{CO}_{\mathrm{z}}$ und $0,1732 \mathrm{~g} \mathrm{H}_{2} \mathrm{O}$.

2. $0,2140, ", 0,4584, ", \quad, 0,1052,, "$

Gefunden:

1.2 . 2.

C $\quad 58,41 \quad 58,41$

$\mathrm{H} \quad 5,57 \quad 5,45$

Berechnet für

$\mathrm{C}_{22} \mathrm{H}_{24} \mathrm{O}_{10}$ :

$58,80 \%$

5,33,

Aus den ermittelten Zahlen lassen sich verschiedene Formeln berechnen. Die oben aufgestellte Formel ergab sich als richtig durch die Untersuchung der Spaltungsprodukte (s. u.).

Krystall wasserbestim mung. $0,2040 \mathrm{~g}$ lufttrockenes, wasserhaltiges Sakuranin vorloren beim Trocknen bei $100^{\circ} 0,0285 \mathrm{~g}$ Wasser.

$\begin{array}{cc}\text { Gefunden: } & \text { Berechnet für } \mathrm{C}_{22} \mathrm{H}_{24} \mathrm{O}_{10}+4 \mathrm{H}_{2} \mathrm{O} \text { : } \\ \mathrm{H}_{2} \mathrm{O} \quad 13,9 & 13,8 \%\end{array}$

\section{Einwirkung von Essigsäureanhydrid auf Sakuranin.}

Tetraacetylsakuranin: $\mathrm{C}_{22} \mathrm{H}_{20}\left(\mathrm{C}_{2} \mathrm{H}_{3} \mathrm{O}\right)_{4} \mathrm{O}_{10}$. $2 \mathrm{~g}$ Sakuranin wurden in einem Kölbchen mit $20 \mathrm{ccm}$ Essigsäureanhydrid versetzt und 2 Stunden lang in gelindem Sieden gehalten. Nach dem Erkalten wurde das Reaktionsprodukt in kaltes Wasser eingegossen, die hierbei ausgeschiedene klebrige Masse alsdann mit kaltem Wasser gut gewaschen, hierauf in Alkohol gelöst und die alkoholische Lösung in viel kaltes Wasser eingetragen. Es entstand ein käsiger Niederschlag, welcher nach dem Trocknen eine gelblich gefärbte, amorphe Masse bildete. Derselbe ist leicht löslich in Alkohol, Chloroform und Benzol, unlöslich in kaltem Wasser, Petroleumbenzin und kalter Alkalilauge. Die zahlreichen 
Bemühungen, diese Substanz zur Krystallisation zu bringen, waren resultatlos. Zur Analyse benutzte ich die Substanz, welche in folgender Weise gereinigt war: Ich löste die Substanz in wenig Alkohol und goß die Lösung in viel Wasser ein. Diese Operation wurde dreimal wiederholt. Die so gereinigte Substanz bildete ein hellgelbes Pulver, erweichte bei $70^{\circ}$ und schmolz gegen $80^{\circ}$ zu einer zähen Masse.

$0,3927 \mathrm{~g}$ im Exsikkator über Schwefelsüure getrocknete Substanz ergaben $0,8.504 \mathrm{~g} \mathrm{CO}_{2}$ und $0,1958 \mathrm{~g} \mathrm{H}_{2} \mathrm{O}$.

\section{Berechnet für}

\begin{tabular}{lrrrrr} 
Gefunden: & Mono- & \multicolumn{2}{c}{ Di- } & Tri- & Tetra- \\
& & \multicolumn{4}{c}{ acetylsakuranin: } \\
C & $\mathbf{5 8 , 5 2}$ & $\mathbf{5 8 , 7 7}$ & $\mathbf{5 8 , 6 6}$ & $\mathbf{5 8 , 6 4}$ & $\mathbf{5 8 , 4 4 \%}$ \\
H & $\mathbf{5 , 3 4}$ & $\mathbf{5 , 3 0}$ & $\mathbf{5 , 2 6}$ & $\mathbf{5 , 2 2}$ & $\mathbf{5 , 1 9 , "}$
\end{tabular}

Zur Kontrolle wurde die Acetylzahl bestimmt. 0,2006 g Substanz wurden in $50 \mathrm{ccm}$ Alkohol (gereinigt durch Destillation über Aetzkali) gelöst, die Lösung mit $1 \mathrm{~g}$ Kaliumhydroxyd versetzt und auf dem Wasserbade 1 Stunde lang erwärmt. Dann wurde das Gemisch durch Verdampfen von Alkohol befreit, mit offizineller Phosphorsäure angesäuert und der Dampfdestillation unterworfen, bis noch ein saures Destillat ïberging. Das etwa 11 betragende Destillat wurde mit $1 / 10$ Normalkalilösung, unter Anwendung von Rosolsäure. als Indikator, titriert. Es wurden zum Neutralisieren $13,0 \mathrm{ccm}$ Lauge verbraucht.

$$
\begin{aligned}
& \text { Gefunden: Berechnet für } \mathrm{C}_{22} \mathrm{H}_{20}\left(\mathrm{C}_{2} \mathrm{H}_{3} \mathrm{O}\right)_{4} \mathrm{O}_{10} \text { : } \\
& \mathrm{CH}_{3} \mathrm{COOH} \quad 38,88 \\
& 38,96 \%
\end{aligned}
$$

Die Acetylierung wurde auch unter Zusatz von Natriumacetat ausgeführt. Hierbei wurde ebenfalls ein amorphes, gegen $80^{\circ}$ schmelzendes Pulver erhalten. Die Farbe desselben war aber diesmal rein weiß. Eine Analyse wurde davon nicht ausgeführt.

\section{Einwirkung von Benzoylchlorid auf Sakuranin.}

Tetrabonzoy lsakuranin: $\mathrm{C}_{22} \mathrm{H}_{20}\left(\mathrm{C}_{7} \mathrm{H}_{5} \mathrm{O}\right)_{4} \mathrm{O}_{10} . \quad 2 \mathrm{~g}$ Sakuranin wurden in $30 \mathrm{ccm}$ Pyridin gelöst und diese Lösung mit $10 \mathrm{ccm}$ Benzoylchlorid versetzt. Sofort erwärmte sich das Gemisch unter Rotfärbung und Abscheidung von salzsaurem Pyridin. Nach dem Erkalten wurde es in Wasser eingegossen. Die hierbei ausgeschiedene rötlich gefärbte Masse erstarrte beim Reiben mit einem Glasstab krystallinisch. Zur Reinigung wurde das getrocknete Reaktionsprodukt in Chloroform heiß gelöst und das Filtrat mit 
Petroleumbenzin gefällt. Diese Operation wurde zweimal wiederholt. Die so gereinigte Substanz bildete ein weißes, unter dem Mikroskop warzenförmig krystallisiertes Pulver. Es ist ziemlich leicht löslich in Chloroform, schwer löslich in Alkohol, Aether, Essigäther und Benzin. In kalter Alkalilauge ist es unlöslich. Beim Erhitzen in einom Kapillarrohr sintert es gegen $220^{\circ}$ zusammen und schmilzt vollständig bei $227^{\circ}$. Beim Eintragen in rauchende Salpetersäure färbt es sich anfangs orangegelb, dann geht es in blaue Tropfen über.

$0,2251 \mathrm{~g}$ bei $100^{\circ}$ getrocknete Substanz ergaben $0,5748 \mathrm{~g} \mathrm{CO}_{2}$ und $0,0962 \mathrm{~g} \mathrm{H}_{2} \mathrm{O}$.

\begin{tabular}{|c|c|}
\hline Gefunden: & Berechnet für $\mathrm{C}_{22} \mathrm{H}_{20}\left(\mathrm{C}_{7} \mathrm{H}_{3} \mathrm{O}\right)_{1} \mathrm{O}_{10}$ : \\
\hline C $\quad 69,63$ & $69,44 \%$ \\
\hline H $\quad 4,74$ & 4,62, \\
\hline
\end{tabular}

\section{Hydrolytische Spaltung des Sakuranins.}

A. Sakuranetin: $\mathrm{C}_{16} \mathbf{H}_{14} \mathbf{O}_{5}$.

Je $5 \mathrm{~g}$ Sakuranin wurden mit $120 \mathrm{ccm}$ verdünnter Schwefelsäure $(5 \%)$ etwa $1 / 2$ Stunde lang gekocht. Es entstand hierdurch ein gelblich gefärbtor, öliger Niederschlag, welcher bald krystallinisch erstarrte. Derselbe wurde abfiltriert und mit Wasser gut ausgewaschen. Das getrocknete, gelb bis braun gefärbte Spaltungsprodukt wurde alsdann in Acther gelöst und diese Lösung mit 'Tierkohle einige Zeit digeriert. Die farblose ätherische Lösung wurde hierauf durch freiwilliges Verdunsten der Krystallisation üborlassen. Zur weiteren Reinigung wurden die Krystalle nochmals aus Aether oder besser aus Benzol umkrystallisiert.

Das reine Spaltungsprodukt, welches ich $S$ ak u $r$ anetin nennen will, bildet farblose, geruch- und geschmacklose Nadeln; dasselbe ist leicht löslich in Alkohol, Aether, Chloroform, Benzol, Essigäther und Pyridin, schwer löslich in kochendem Wasser, fast unlöslich in kaltem Wasser. Aus Aether oder Benzol krystallisiortes Sakuranetin ist wasserfrei und schmilzt bei $150^{\circ}$. Aus kochendem Wasser umkrystallisiertes oder aus alkoholischer Ijösung durch Wasserzusatz ausgefälltes Sakuranetin stellt feine, filzige Nadeln dar, welche wasserhaltig sind und gegen $70^{\circ}$ unter Abgabe von Krystallwasser schmelzen. Durch Ammoniak und durch Alkalien wird das Sakuranetin leieht gelöst und aus diesen Lösungen durch verdünnte Säuren, wie auch durch Kohlensäure, wieder abgeschieden. Die alkalische Lösung des Sakuranetins ist intensiv gelb gefärbt. Rauchende Salpetersäure gibt sofort eine tief indigoblaue Färbung, 
welche allmählich in Violett übergeht. Mit Natriumamalganı und Wasser erhitzt, bildet Sakuranetin eine Substanz, welche daraus durch Salzsäure gefällt werden kann. Der Niederschlag löst sich in Alkohol mit roter Farbe. Die Lösung zeigt aber keine Fluorescenz.

$$
\text { Analyse des Sakuranetins. }
$$

Bei $100^{\circ}$ getrocknetes Sakuranetin wurde in einem geschlossenen Rohre mit Kupferoxyd verbrannt.

1. $0,4040 \mathrm{~g}$ lieferten $0,9923 \mathrm{~g} \mathrm{CO}_{2}$ und $0,1860 \mathrm{~g} \mathrm{H}_{2} \mathrm{O}$.

2. $0,2758, ", 0,6776, ", \quad, \quad 0,1272, \quad$,

3. $0,2124, ", 0,5215, ", \quad, 0,1028,, "$,

\begin{tabular}{ccccc} 
& \multicolumn{3}{c}{ Gefunden: } & Berechnet für \\
& 1. & 2 & 3. & $\mathrm{C}_{16} \mathrm{H}_{14} \mathrm{O}_{5}:$ \\
$\mathrm{C}$ & 66,98 & 67,00 & 66,95 & $67,13 \%$ \\
$\mathrm{H}$ & 5,10 & 5,14 & 5,37 & $4,90 .$,
\end{tabular}

Krystallwasserbestimmung. $0,2080 \mathrm{~g}$ lufttrockenes, wasserhaltiges Sakuranetin verloren bei $100^{\circ}$ getrocknet $0,0224 \mathrm{~g}$ Wasser.

$$
\begin{array}{cc}
\text { Gefunden: } & \text { Berechnet für } \mathrm{C}_{16} \mathrm{H}_{14} \mathrm{O}_{5}+2 \mathrm{H}_{2} \mathrm{O}: \\
\mathrm{H}_{2} \mathrm{O} \quad 10,74 & 11,0 \%
\end{array}
$$

\section{B. Traubenzucker.}

Das Filtrat vom Sakuranetin reduzierte stark Fe h ling' sche Lösung und lenkte das polarisierte Licht nach rechts ab. Es wurde durch Baryumkarbonat von Schwefelsäure befreit und zur Sirupkonsistenz eingedampft. Der süß schmeckende Rückstand erstarrte nach mehreren Tagen zu einer undurchsichtigen Krystallmasse. 1 Teil dieser Masse wurde mit 3 Teilen Natriumacetat und 2 Teilen salzsaures Phenylhydrazin in 20 Teilen Wasser kalt gelöst und mehrere Stunden lang in der Kälte stehen gelassen. Es entstand keine Fällung. Danñ wurde das Gemisch mit Essigsäure stark angesäuert und auf dem Wasserbade erwärmt. Alsbald erschien eine reichliche Abscheidung von gelben Nadeln, welche abgesogen und getrocknet wurden. Zur Prüfung auf Rhamnoseosazon wurde dieselbe mit Aceton digeriert. Die gelb gefärbte Acetonlösung hinterlie $ß$ beim Verdampfen eine kleine Menge brauner Substanz, welche gegen $200^{\circ}$ unter Zersetzung schmolz. Also wurde kein Rhamnoseosazon (Schmp. 180 ${ }^{\circ}$ ) nachgewiesen. Die in Aceton unlösliche Substanz gab, nach der Umkrystallisation aus kochendem $50 \%$ igem Alkohol, bei $204^{\circ}$ schmelzende Nadeln. Beim Oxydieren des Zuckers mittelst verdünnter Salpetersäure erhielt ich nur eine sirupöse, in Wasser leicht lösliche Masse. Die 
in Wasser schwer löslichen sandigen Krystalle von Schleimsäure sind nicht beobachtet worden.

Aus diesen Beobachtungen geht hervor, daß der Zucker, welcher durch Spaltung von Sakuranin gebildet wird, nur aus d-Glykose besteht.

\section{Quantitative Bestimmung des Sakuranetins.}

Die Spaltung des Sakuranins vollzieht sich im Sinne folgender Gleichung:

$$
\mathrm{C}_{22} \mathrm{H}_{24} \mathrm{O}_{10}+\mathrm{H}_{2} \mathrm{O}=\mathrm{C}_{16} \mathrm{H}_{14} \mathrm{O}_{5}+\mathrm{C}_{8} \mathrm{H}_{12} \mathrm{O}_{6} \text {. }
$$

Nach dieser Gleichung müssen 100 Teile Sakuranin 63,6 Teile Sakuranetin liefern. Zur Prüfung desselben wurde $1 \mathrm{~g}$ Sakuranin (wasserfrei) mit $100 \mathrm{ccm} 1 \%$ iger Schwefelsäure in einem Kölbchen $1 / 2$ Stunde lang gekocht und das erkaltete Gemisch mit gleichem Volumen Aether zweimal ausgeschüttelt. Die vereinigte ätherische lösung wurde in einem weithalsigen tarierten Kolben der Destillation unterworfen, und der Rückstand schließlich bei $100^{\circ}$ zum konstanten Gewicht getrocknet.

Es bliob 0,6401 $\mathrm{g}$ wasserfreies Sakuranetin zurück:

$\begin{array}{cc}\text { Gefunden: } & \text { Berechnet: } \\ 64,0 & 63,6 \%\end{array}$

\section{Einwirkung von Essigsãureanhydrid auf Sakuranetin.}

$2 \mathrm{~g}$ Sakuranetin wurden mit $2 \mathrm{~g}$ geschmolzenem Natriumacetat und $20 \mathrm{ccm}$ Essigsäureanhydrid 2 Stunden lang in einem Paraffinbade gekocht. Nach dem Erkalten wurde das Gemisch in Wasser eingegossen. Die hierbei ausgeschiedene sirupöse Masse war leicht löslich in Alkohol und Aether, unlöslich in Wasser und kalter Lauge. Trotz mehrerer Versuche mit verschiedenen Lösungsmitteln war diese Substanz nicht krystallinisch zu erhalten. Selbst nach monatelangem Aufbewahren im Exsikkator ließ sie sich nicht pulverisieren.

\section{Elnwirkung von Benzoylchlorid auf Sakuranetin.}

Monobenzoylsakuranetin: $\mathrm{C}_{16} \mathrm{H}_{13}\left(\mathrm{C}_{7} \mathrm{H}_{5} \mathrm{O}\right) \mathrm{O}_{5}$. $2 \mathrm{~g}$ Sakuranetin wurden in $30 \mathrm{ccm}$ Pyridin gelöst und diese Lösung mit $10 \mathrm{ccm}$ Benzoylchlorid auf einmal versetzt. Sofort erwärmte sich die Mischung unter Rotfärbung und Abscheidung von salzsqurem Pyridin. Nach dem Erkalten wurde das Produkt in kaltes Wasser eingegossen. Der rotbraune Niederschlag wurde in Chloroform gelöst und die Lösung mit Petroleumbenzin gefällt. 
Der Niederschlag bestand aus einem weißen, krystallinischen Pulver vom Schmp. 170". Es ist leicht löslich in Chloroform, etwas löslich in warmem Fisessig, sehr schwer löslich in Alkohol, Aether, Essigäther und Benzin. In Wasser und kalter Lauge ist es unlöslich. Tropfen.

Rauchende Salpetersäure gibt nach einiger Zeit hellblaue

Die Elementaranalysen der bei $100^{\circ}$ getrockneten Substanz. ergaben folgende Daten:

1. $0,2324 \mathrm{~g}$ lieforten $0,6014 \mathrm{~g} \mathrm{CO}_{2}$ und $0,0950 \mathrm{~g} \mathrm{H}_{2} \mathrm{O}$.

2. $0,2052, ", 0,5336, ", \quad, 0,0862,, \quad$, Gofunden: Berechnet für

\begin{tabular}{|c|c|c|c|}
\hline & 1. & 2. & $\mathrm{C}_{18} \mathrm{H}_{13}\left(\mathrm{C}_{7} \mathrm{H}_{5} \mathrm{O}\right) \mathrm{O}_{5}$ : \\
\hline C & 70,56 & 70,91 & $70,76 \%$ \\
\hline $\mathbf{H}$ & 4,48 & 4,66 & 4,76, \\
\hline
\end{tabular}

Methoxylbestimmung des Sakuranetins.

Die Methoxylbestimmungen des Sakuranetins wurden nach $\mathrm{Z}$ e is e 1 ausgeführt und ergaben folgende Resultate:

1. 0,3922 g Sakuranetin (wasserfrei) ergaben $0,3128 \mathrm{~g} \mathrm{AgJ}$.

2. $0,4462, " \quad$,

3. 0,2406 ,
Gefunden:
1. 2.3.
$\mathrm{CH}_{3} \mathrm{O} \quad 10,5 \quad 10,4 \quad 10,4$
Berechnet für
$\mathrm{C}_{15} \mathrm{H}_{11}\left(\mathrm{CH}_{3} \mathrm{O}\right) \mathrm{O}_{4}$ :
$10,8 \%$

$0,3547,,$,

$0,1912,,$, handen.

Im Sakuranetinmolekiil ist somit eine Methoxylgruppe vor-

\section{Einwirkung von Brom auf Sakuranetin.}

Wird Sakuranetin in Chloroform gelöst, und diese Lösung mit einer Chloroformlösung von Brom tropfenwcise versetzt, so wird die Mischung anfangs rasch entfärbt. Wenn aber die luösung eine bleibende braune Farbe annimmt und die Entwickelung von Bromwasserstoff bemerkbar wird, so findet die Abscheidung von gelblich gefärbten prismatischen Krystallen statt. Dieselben sind sehr schwer löslich in den gewöhnlichen Lösungsmitteln (Alkohol, Aether, Chloroform, Essigäther etc.). Kochender Eisessig nimmt etwas davon auf. Zur Reinigung wurden die Krystalle in viel heißem Eisessig gelöst und das Filtrat durch Wasserzusatz ausgefällt. Der so orhaltene Niederschlag bildet gelblich weiße, zu beiden Enden zugespitzte und in der Mitte kugelig angeschwollene, feine Nadeln vom Schmp. 217 . Die Menge dieses Bromderivats reichte leider zur Analyse nicht aus. 


\section{Kallschmelze des Sakuranetins}

$10 \mathrm{~g}$ Sakuranetin wurden mit $50 \mathrm{~g}$ Kaliumhydroxyd in wenig Wasser gelöst und eine Stunde lang unter stetigem Umrühren erhitzt, wobei die Temperatur allmählich bis auf $200^{\circ}$ gesteigert wurde. Nach dem Erkalten wurde die Schmelze in etwa $100 \mathrm{ccm}$ Wasser gelöst und die Lösung filtriert. Die Lösung wurde mit verdünnter Schwefelsäure schwach angesäuert, ein sehr kleiner Ueberschuß von Kaliumbikarbonat zugefügt und dreimal mit reichlicher Menge Aether $(A)$ ausgeschüttelt. Hicrbei entstand aus der wässerigen Lösung eine reichliche Abscheidung von Kaliumsulfat. Dasselbe wurde abfiltriert und die Lösung $(B)$ wurde zur weiteren Verarbeitung beiseite gelegt.

1. Phloroglu c in. Die ätherische Lösung $(A)$ wurde abdestilliert und der Rückstand aus heißem Wasser umkrystallisiert. Die so gewonnenen, rötlich gefärbten Krystalle schmolzen bei $207^{\circ}$ und schmeckten süß. Die wässerige Lösung der Krystalle wird durch Eisenchlorid blauviolett gefärbt. Werden die Krystalle mit Salzsäure auf einen Fichtenspan gebracht, so wird eine rotviolette Färbung hervorgerufen. Mit Vanillin und Salzsäure erhitzt, lieferten die Krystalle eine rote Färbung. Diese Reaktionen stimmen mit denen des Phloroglucins überein. Da die Menge der Krystalle sehr gering war, wurde die Identifizierung durch die Nitroverbindung bewerkstelligt.

$0,2 \mathrm{~g}$ dieser Krystalle wurden in wenig konzentrierter Schwefelsäure gelöst und die Iösung wurde in eine kalte Mischung von je $2 \mathrm{ccm}$ konzentrierter Schwefelsäure und Salpetersäure eingetragen. Nach einigen Minuten wurde das Gemisch in $20 \mathrm{ccm}$ kaltes Wasser eingegossen. Die ausgeschiedenen Krystalle wurden abfiltriert, mit Salzsäure gewaschen und bei $100^{\circ}$ getrocknet. Dieselben schmolzen bei $165^{\circ}$ (wasserfreies Trinitrophloroglucin schmilzt bei $167^{\circ}$ ).

2. Essigsä u r e. Die Lösung $(B)$ wurde mit Schwefelsäure von neuem stark angesäuert und der Dampfdestillation unterworfen. Es ging ein saures Destillat über, welches mit Soda neutralisiert und eingedampft wurde. Eine Probe desselben gab mit Fisenchlorid eine gelblich-rote Färbung. Der ganze Rest wurde mit Silbernitratlösung versetzt und der anfangs weiße, bald in Grauviolett übergehende Niederschlag abfiltriert und mit Wasser ausgewaschen. Derselbe wurde aus kochendem Wasser umkrystallisiert, wobei eine starke Reduktion von Silber eintrat. Das reine Silbersalz bildete glänoende, durchscheinende Nadeln. 
$0,1593 \mathrm{~g}$ bei $100^{\circ}$ getrockneter Substanz hinterließen beim Glühen $0,1032 \mathrm{~g}$ Silber.
Gefunden:
Ag $\quad 64,78$
Berechnet für $\mathrm{CH}_{3} \mathrm{COOAg}$ :
$64,66 \%$

3. $\mathrm{p}-\mathrm{O} \times \mathrm{y}$ b e n z oes ä u re. Die von Essigsäure befreite saure Lösung wurde wiederholt mit Aether ausgeschüttelt. Die ätherische Lösung hinterließ beim Verdampfen einen krystallinischen Rückstand. Derselbe wurde aus kochendem Wasser umkrystallisiert. Die so gereinigte Substanz bildete weiße, prismatische Nadeln vom Schmp. $210^{\circ}$. Die wässerige Lösung wird durch Eisenchlorid gelblich braun gefärbt.

Die Elementaranalyse und die Silberbestimmung ergaben folgende Daten:

$0,1906 \mathrm{~g}$ bei $100^{\circ}$ getrocknete Substanz lieferten $0,4216 \mathrm{~g} \mathrm{CO}_{2}$ und $0,0804 \mathrm{~g} \mathrm{H}_{2} \mathrm{O}$.

$0,2572 \mathrm{~g}$ bei $100^{\circ}$ getrocknetes Silbersalz ergaben beim Glühen $0,1122 \mathrm{~g}$ Silber.
Gefunden:
C 60,32
H 4,61
Berechnet für $\mathrm{C}_{6} \mathrm{H}_{4}(\mathrm{OH}) \mathrm{COOH}$ :
Gefunden:
Ag 43,63
$66,86 \%$
4,34,
Berechnet für $\mathrm{C}_{6} \mathrm{H}_{4}(\mathrm{OH}) \mathrm{COOAg}$ :
$44,08 \%$

\section{Einwirkung von Kalilauge auf Sakuranetin.}

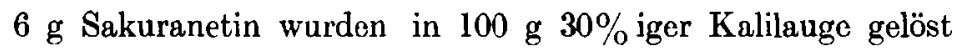
und in einem weithalsigen Kölbchen bis zur Sirupkonsistenz kochend eingedampft. Nach dem Erkalten wurde der Krystallbrei mit Wasser verdünnt, mit verdünnter Schwefelsäure schwach angesäuert, dann mit Kaliumbikarbonat wieder alkalisch gemacht und mit Aether ausgeschüttelt. Die ätherische Lösung hinterließ beim Verdampfen einen braunen, sirupartigen Rückstand. Derselbe zeigte charakteristische Fichtenspanreaktion, aber konnte nicht im krystallisierten Zustand erhalten werden.

Die wässerige Lösung wurde mit Schwefelsäure stark angesüuert und wiederum durch Ausschütteln mit Aether ausgezogen. Beim Verjagen des Aethers blieb ein stark nach Essigsäure riechender, sirupöser Rückstand, welcher beim Umrühren mit einem Glasstab größtenteils krystallinisch erstarrte. Durch Umkrystallisation aus heißem Wasser erhielt ich gelbliche, prismatische Krystalle, welche bei $206^{\circ}$ schmolzen und mit Eisenchlorid eine gelbliche Farbenreaktion gaben 
Sowohl durch Elementaranalyse, als auch durch Silberbestimmung des Silbersalzes, haben diese Krystalle sich als p - O x y benz o e s ä u re erwiesen.

$0,1592 \mathrm{~g}$ bei $100^{\circ}$ getrockneter Substanz lieferten $0,3547 \mathrm{~g} \mathrm{CO}_{2}$ und $0,0554 \mathrm{~g} \mathrm{H}_{2} \mathrm{O}$.

$0,1200 \mathrm{~g}$ Silbersalz hinterließen beim Glühen $0,0526 \mathrm{~g}$ Silber.

Gefunden:

C $\quad 60,76$

H 3,85

Gefunden:

$\mathrm{Ag} \quad 43,8$
Berechnet für $\mathrm{C}_{8} \mathrm{H}_{4}(\mathrm{OH}) \mathrm{COOH}$ :

$80,86 \%$

4,34,

Berochnet für $\mathrm{C}_{8} \mathrm{H}_{4}(\mathrm{OH}) \mathrm{COOAg}$ :

$44,08 \%$

Aus Mangel an Material kann ich jetzt die Untersuchung nicht weiter verfolgen. Sobald größere Mengen dieser Rinde erhältlich scin werden, soll die Untersuchung, namentlich über die Konstitution des Sakuranetins, ron neuem in Angriff genommen werden.

\section{Zusammenstellung und Bemerkungen.}

1. Die Rinde von Prunus Pseudo-Cerasus var. Sieboldi enthält ein Glykosid von der Formel $\mathrm{C}_{22} \mathrm{H}_{24} \mathrm{O}_{10}$, welches $\mathrm{S}$ a k u r a n i n genannt wird.

2. Sakuranin liefert ein Tetraacetyl- und Tetrabenzoylderivat, und wird beim Kochen mit verdünnten Säuren in $\mathrm{Sakuranet}$ i $\mathrm{C}_{16} \mathrm{H}_{14} \mathrm{O}_{5}$ und Traubenzucker gespalten. Die Spaltung vollzieht sich im Sinne der folgenden Gleichung:

$$
\mathrm{C}_{22} \mathrm{H}_{24} \mathrm{O}_{10}+\mathrm{H}_{2} \mathrm{O}=\mathrm{C}_{18} \mathrm{H}_{14} \mathrm{O}_{5}+\mathrm{C}_{6} \mathrm{H}_{12} \mathrm{O}_{8} \text {. }
$$

3. Sakuranetin liefert ein amorphes Acetylderivat und eine krystellisierte Monobenzoylverbindung. Es enthält eine Methoxylgruppe; durch Kalischmelze wird das Sakuranetin in Phloroglucin, Essigsäure und p-Oxybenzoesăure gespalten. Kochende Alkalilauge liefert ebenfalls p-Oxybenzoesäure, Essigsäure (?) und ein nicht näher charakterisiertes Phloroglucinderivat.

4. Die Konstitution des Sakuranetins ist nicht sichergestellt; wahrscheinlich ist Sakuranetin eine dem Cotoin ähnliche Verbindung oder ein Phloroglucid, welches dem Phloretin, dem Naringenin und dem Hesperetin nahe steht.

Zuerst glaubte ich, daß das Sakuranetin mit dem Monomethylnaringenin identisch sei. Bekanntlich liefert Naringenin $\left(\mathrm{C}_{15} \mathrm{H}_{12} \mathrm{O}_{5}=\right.$ p-Cumarsäurephloroglucid $)$ durch Natriumamalgam 
eine Substanz, welche sich in Alkohol mit roter Farbe löst. Dies ist auch der Fall bei dem Sakuranetin. Da aber Sakuranetin beim Kochen mit Kalilauge nicht p-Cumarsäure sondern p-Oxybenzoesäure gibt, so bedarf diese Vermutung noch weiterer Bestätigung.

5. Herr Prof. Dr. Ta $\mathrm{k}$ a h a s h i hat die Güte gehabt, eine Reihe von pharmakologischen Versuchen mit dem. Sakuranin auszuführen. Dafür spreche ich ihm meinen besten Dank aus. Es ergab sich, daß dieses Glykosid physiologisch unwirksam ist und entgegen dem Phloridzin Glykosurie nicht verursachen kann.

6. Im Laufe dieser Untersuchung nahm ich Gelegenheit auch die Rinde von Prunus Miqueliana Maxim. zu prüfen, aber konnte darin kein Sakuranin nachweisen.

Dem Herrn Prof. Dr. Sh i m oy a m a spreche ich für den mir zuteil gewordenen Rat meinen aufrichtigen Dank aus.

Toky o, den 29. Februar 1908.

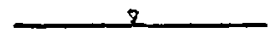

\title{
Aus dem Laboratorium fur galenische Pharmazie der Universität Paris. \\ Von Professor Dr. Em. B o u r qu elot.
}

\section{Ueber das Verbenalin, das Glykosid der Verbena officinalis $\mathrm{L}$.}

\author{
Von L. B o urdier.
}

(Eingegangen den 25. III. 1908.

Die Alten haben der Verbene verschiedene Eigenschaften zugeschrieben: ihr Aufguß sollte die Blähungen vertreiben und die Kolik beseitigen; ihr destilliertes Wasser sollte Augenkrankheiten, Geschwüre des Mundes, Wassersucht und Bleichsucht heilen, sowie dic Milch der Ammen vermehren.

Neben diesen therapeutischen Eigenschaften teilte man der Verbene auch gleichzeitig magische zu. Die Zauberer bedienten sich daher derselben; die Griechen fertigten daraus Kronen für die Wappenherolde; die Priester gebrauchten sie, um die Altäre vor den Opfern zu reinigen; die Druiden pflückten dieselben mit besonderen Zeremonien: sie brachten zuvor der Erde ein Opfer und rissen sie nur bei Beginn des Tages aus. 